Revue d'histoire de l'Amérique française

DEV RUE D.HISTOIRE DE L'AMÉRIQUE FRANÇAISE

\title{
L'importance stratégique des colonies antillaises dans la politique française de l'après-guerre (1763-1770)
}

\section{R. John Singh}

Volume 28, numéro 1, juin 1974

URI : https://id.erudit.org/iderudit/303327ar

DOI : https://doi.org/10.7202/303327ar

Aller au sommaire du numéro

Éditeur(s)

Institut d'histoire de l'Amérique française

ISSN

0035-2357 (imprimé)

1492-1383 (numérique)

Découvrir la revue

Citer cet article

Singh, R. J. (1974). L'importance stratégique des colonies antillaises dans la politique française de l'après-guerre (1763-1770). Revue d'histoire de l'Amérique française, 28(1), 27-43. https://doi.org/10.7202/303327ar d'utilisation que vous pouvez consulter en ligne. 


\title{
L'IMPORTANCE STRATEGIQUE DES COLONIES ANTILLAISES DANS LA POLITIQUE FRANÇAISE DE L'APRĖS-GUERRE 1763.1770
}

\author{
R. John SingH \\ Département d'histoire \\ Kearney State College \\ Nebraska, Etats-Unis
}

Les études traitant de la politique étrangère française après la guerre de Sept ans mettent communément en relief le fait que le principe directeur des relations franco-anglaises était la préparation d'une guerre qui vengerait la défaite subie lors du Traité de Paris, en 1763. Elles montrent clairement que la France n'avait pas accepté le démembrement de son empire, la perte de son prestige en Europe, et son humiliation dans le monde colonial. Les historiens reconnaissent que les ministres français commencèrent très tôt à préparer un plan de représailles qui mobiliserait toutes les forces françaises, qu'elles fussent du continent ou des colonies. Ils affirment, en outre, qu'un tel plan se fondait sur l'espoir de renforcer le Pacte de Famille avec l'Espagne et de venger la défaite par l'union des couronnes bourboniennes. ${ }^{1}$

Mais ces études sont insuffisantes parce qu'elles ne reconnaissent pas, comme elles le devraient, l'importance du rôle joué par les colonies françaises dans la mise en œuvre du plan de guerre français. Les colonies contribuèrent à justifier la guerre et à fournir les moyens de la faire. Par leur situation unique, elles offraient un grand avantage à la France puisqu'elles permettaient de frapper directement et efficacement les colonies anglaises, engageant, de ce fait, une guerre coloniale et maritime.

1 Il existe un certain nombre de bons ouvrages traitant de la politique étrangère française, mais ils ne contiennent pas la thèse épousée dans cette étude. Voir: John F. Ramsey, Anglo-French Relations, 1763-1770: A study of Choiseul's foreign policy (Berkeley, 1939), C. L. Lokke, France and the colonial question: A study of contemporary French opinion 1763-1801 (New York, 1932), Alfred Bourget, Etudes sur la politique étrangère du Duc de Choiseul (Paris, 1907), E. Daubigny, Choiseul et la France d'outre mer après le traité de Paris (Paris, 1892).

RHAF, vol. 28, no 1 (juin 1974) 
Aussitôt après la guerre de Sept ans, Louis XV n'était pas disposé à se lancer dans une autre guerre européenne, mais les ministres français le convainquirent que tout conflit à venir devait commencer dans les colonies, qu'elles en partageraient une juste part du coût et que, d'après un programme amendé par le ministère, les régiments coloniaux renforceraient considérablement les bataillons français de la métropole. Ils le persuadèrent aussi que, avec l'aide des forces espagnoles, liées par le Pacte de Famille, la France remporterait la victoire sur l'Angleterre et les colonies anglaises. Ils donnèrent, en plus, comme arguments, que ces avantages et la stratégie de combat par diversion assureraient la réparation de la défaite subie lors du Traité de Paris.

Etant donné ces circonstances, il est permis de se demander pourquoi ces études ont négligé de souligner l'influence et l'importance des colonies dans l'élaboration de la stratégie de guerre de la France peu après 1763. Peut-être pourrait-on soutenir la thèse que les historiens du $18 \mathrm{e}$ et du début du $19 \mathrm{e}$ siècle étaient captifs du jeu des intérêts de l'expansion européenne dans le Nouveau-Monde et de la rivalité des colonies à l'âge de l'impéralisme. Ils croyaient qu'elles n'existaient que pour servir l'intérêt des pays métropolitains et qu'elles constituaient essentiellement des centres d'échange et de commerce. Les ouvrages d'Edward Long, History of Jamaica (1774), de Bryan Edwards, History civil and commercial of the British colonies in the West Indies (1793), et de l'abbé Raynal, Histoire philosophique et politique des établissements et du commerce des Européens dans les deux Indes (1780), illustrent bien cette thèse.

Les historiens contemporains, à quelques exceptions près, se rangent dans la même tradition. Cependant celle-ci est de plus en plus contestée par une nouvelle approche du sujet dont nous trouvons un exemple dans les ouvrages d'Eric Williams, Negro in the Caribbean (1942), Capitalism and Slavery (1961), et British Historians in the West Indies (1966). Les travaux de Williams, écrits dans le même esprit que l'Abolition of the African slave Trade (1830) de Thomas Clarkson, et l'ouvrage plus ancien d'Adam Smith, Wealth of Nation (1776), ont d'ailleurs suscité un intérêt considérable pour les études sur les Antilles.

Influencé par ce nouveau courant historiographique voulant que les Antilles aient contribué de façon significative à la construction de l'histoire européenne, l'auteur de ces pages a pu trouver confirmation de cette hypothèse dans les dépôts d'archives 
françaises. Il a consigné les résultats de ses recherches dans une thèse de doctorat présentée à l'Université de l'Oklahoma et qui sera bientôt publiée. Il ne donne ici qu'un résumé de ses découvertes. Il énumère les considérations qui incitèrent le duc de Choiseul, ministre de la Marine, de la Guerre et des Affaires étrangères, à formuler une nouvelle politique étrangère, qui transforma la diplomatie française. Les lecteurs seront en mesure de juger par eux-mêmes, grâce aux preuves documentaires à l'appui, de la validité de sa thèse.

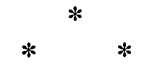

Etienne-François de Choiseul fut l'architecte de la nouvelle politique française élaborée après 1763 . Il croyait qu'une confrontation militaire avec l'Angleterre était le seul moyen d'assurer le redressement de la situation résultant de la défaite subie lors de la guerre de Sept ans en même temps que d'assurer la restauration du prestige et de la puissance de la France. Il essaya non seulement de convaincre Louis XV de la nécessité d'une telle offensive mais il commença à élaborer des plans en vue de préparer une guerre de revanche contre l'Angleterre. Il envisagea une réorganisation complète des forces défensives de l'armée, de la marine et de l'artillerie, tant en France que dans les colonies, et le renforcement du Pacte de Famille avec l'Espagne: mesures qui nécessiteraient de grosses sommes d'argent, même avec le soutien des colonies. Et pourtant, à ce moment-là, la France était incapable d'entreprendre cette tâche sans l'appui du parlement et des classes de propriétaires exemptés d'impôts, en vertu de privilèges fiscaux. L'alternative aurait nécessité l'imposition de nouvelles taxes, ce que Choiseul ne pouvait faire, et par ailleurs le faible crédit du gouvernement français rendait difficile d'obtenir de nouveaux prêts.

Choiseul convainquit néanmoins Louis XV que la France n'était pas la seule à éprouver des difficultés financières. $\mathrm{Au}$ dire du ministre la situation de l'Angleterre était pire que celle de la France. Il soutenait que le gouvernement britannique était si accablé de problèmes, à la fois à l'intérieur du pays et dans les colonies d'Amérique, qu'une autre guerre ruinerait sa position financière et conséquemment réduirait sa puissance en Europe et dans le monde colonial. Choiseul était convaincu qu'une autre guerre, non seulement accroîtrait la dette nationale de l'Angleterre, mais finalement, la détruirait en tant que puissance maritime. Il conseilla donc au roi de prendre les moyens nécessaires afin de forcer l'Angleterre à dépenser d'énormes sommes d'argent 
et pour soutenir son armée et pour financer une guerre coûteuse. A cet effet, il écrivit dans son Mémoire à Louis XV, en 1765 :

En attendant, il faut, en politique, se précautionner contre ses desseins dangereux, et, en se défendant contre elle, selon les moyens que j'indiquerai à Votre Majesté, tels que je les ai imaginés, à l'article de la marine, chercher à lui susciter moins des ennemis que des occasions de dépenses. C'est pourquoi la guerre contre le Portugal sera toujours, dans tous les cas, une guerre utile à Votre Majesté quelque événement qui en résulte ... Celui qui est plus prochain et qui procurera une chute nécessaire, est l'immensité des dettes de l'Angleterre. Quand cette puissance rentrera en guerre avec Votre Majesté, quels que soient les événements, l'attention principale qu'elle doit ordonner à ses ministres est d'épargner à la France la dépense, autant qu'il sera possible, en occasionnant aux Anglais la plus forte dépense qu'il se pourra. Je sens la difficulté de ce système; mais je crois en connaître la possibilité, et, si Votre Majesté, sans se laisser entraîner à des dépenses excessives dans les premières années de la guerre, comme il est arrivé presque toujours, se ménage de manière à pouvoir fournir à cette guerre une année de plus que les Anglais ne peuvent y fournir, alors ce ne seront plus les succès qui détermineront la paix, ce sera l'état des finances de chaque royaume, et Votre Majesté sera le maître des conditions. ${ }^{2}$

Choiseul soutint en plus que la position de l'Angleterre était minée non seulement par ses problèmes financiers mais aussi par des troubles croissants dans ses colonies d'Amérique et des difficultés grandissantes dans ses affaires intérieures. Ces difficultés s'aggravant, affirmait-il, conduiraient inévitablement à une révolution américaine que le faible gouvernement de l'Angleterre serait impuissant à conjurer. La prédiction de Choiseul se réalisa finalement, mais pendant l'administration de son successeur, le comte de Vergennes. Dans son Mémoire de 1765, Choiseul déclarait à Louis XV :

L'Angleterre est l'ennemie déclarée de votre puissance et de votre Etat: elle le sera toujours. Son avidité dans le commerce, le ton de hauteur qu'elle prend dans les affaires, sa jalousie de votre puissance et plus que cela les particuliers des différentes cabales qui tour à tour la gouvernent, doivent vous faire présager qu'il se passera encore des siècles avant que de pouvoir établir une paix durable avec cet Etat, qui vise à la suprématie dans les quatre parties du monde. Il n'y aura que

2 Etienne François de Choiseul, Mémoire de Monsieur de Choiseul remis au Roi en 1765 (Paris, Charles Giraud, 1881), 8-9 (Abréviation utilisée ci-dessous pour cet ouvrage: Choiseul, Mémoire). 
la révolution d'Amérique qui arrivera, mais que nous ne verrons vraisemblablement pas, qui remettra l'Angleterre dans l'état de faiblesse où elle ne sera plus à craindre en Europe. ${ }^{3}$

Tout en dépeignant l'Angleterre dans une situation pire que celle où se trouvait la France, le ministre pressait le roi de renforcer le Pacte de Famille avec l'Espagne. ${ }^{4}$ Ce Pacte contracté en 1761 pendant la guerre de Sept ans, comprenait un traité de commerce dont Choiseul se servit pour renouveler et améliorer les accords franco-espagnols.

En effet, pendant les cinq années qui suivirent le Traité de Paris, Choiseul consacra un temps considérable à négocier avec le ministre des Affaires étrangères d'Espagne, le marquis de Grimaldi, pour convenir de relations mutuelles et s'entendre sur une politique étrangère commune vis-à-vis de l'Angleterre. ${ }^{5}$ C'est lors des accords de 1768 et de 1769 que Choiseul réussit à renouveler le Pacte, et tout particulièrement en ce qui concernait le commerce, faisant prévaloir encore une fois l'influence française dans les affaires étrangères espagnoles.

Concurremment, Choiseul pressa Charles III, par l'intermédiaire de l'ambassadeur français à Madrid, le comte d'Ossun, d'entreprendre, en Espagne et dans les colonies espagnoles, des réformes militaires et navales semblables à celles accomplies dans l'armée et la marine françaises. Il essaya de convaincre Charles III de la nécessité d'un engagement militaire contre Georges III pour maintenir l'équilibre des forces en Europe et

3 Ibid., 8.

4 En ce qui concerne le Pacte de famille de 1761, voir A.E.C.P., Espagne: fols. 290-294. Au sujet des négociations pour renforcer le Pacte, voir la correspondance adressée par Choiseul (Versailles) à Ossun (Madrid), 12 mai 1761, S.E.C.P., Espagne, vol. 532: fol. 235. Au sujet des traités de 1768 et 1769, voir A.E.C.P., ibid., vol. 533: fols. 270-285. L'ouvrage de Louis Blart, Les Rapports de la France et de l'Espagne après le Pacte de famille (Paris, 1915) discute de façon approfondie les relations franco-espagnoles. L'article de F.-P. Renault, "Etudes sur le Pacte de famille et la politique coloniale française, 1760-1790", Revue d'histoire des colonies françaises, dixième année (Premier semestre, 1922) est important. La thèse de Soeur Mary Austin Collins, qui n'est pas publiée et est intitulée The reforms of Charles the Third in New Spain in the light of the Pacte de famille (Berkeley, 1927) présente quelque intérêt, quoique son apport soit plutôt narratif et que l'auteur ne donne pas d'interprétation personnelle.

$5 \mathrm{La}$ correspondance entre Choiseul et Grimaldi est conservée aux Archives Nationales, séries $K 144$, no 181 . L'ouvrage de Louis Blart, Les Rapports de la France et de l'Espagne après le Pacte de famille (Paris, Librairie Félix Alcan, 1915) peut être consulté comme source secondaire (Abréviation utilisée ci-dessous pour cet ouvrage: Blart, Les Rapports). 
dans le monde colonial, l'Angleterre étant l'ennemie déclarée de la famille des Bourbons et de l'Europe.

Charles III hésita d'abord à approuver le plan établi par Choiseul pour préparer une guerre de revanche contre l'Angleterre. Cependant, le ministre des Affaires étrangères espagnoles était bien disposé envers Choiseul et, le plus souvent, il coopéra avec lui. Ainsi, voyant que Charles III voulait introduire des réformes générales à la suite de la défaite espagnole lors de la guerre de Sept ans, le marquis de Grimaldi usa de son influence pour l'engager à négocier avec son allié Bourbon, en vue d'arriver à une entente mutuelle et à une politique commune. Les pressions exercées par Grimaldi sur Charles III provenaient en fait de l'influence du ministre français. D'après l'historien F.-P. Renault, cette influence fut décisive et les ministres espagnols soutinrent le plan de guerre de Choiseul. ${ }^{6}$

Pour sa part, Louis Blart affirme que les ministres espagnols n'appuyèrent pas les desseins belliqueux de la France. A ce sujet, il écrit que, à Madrid, les ministres se montrèrent ouvertement hostiles à cette alliance, la nation y étant défavorable; en conséquence, elle ne fut conclue que par quelques hommes d'Etat, le duc de Choiseul et Charles III. Selon Blart, l'accord contenait à la fois des points forts et des points faibles et sa réussite ou son échec devait dépendre des événements. Il émet des doutes quant à la solidité du Pacte de Famille mais il reconnaît qu'il était indispensable à la France en cas de guerre maritime. ${ }^{7}$ Choiseul fit valoir à Grimaldi que si le Pacte de Famille était renforcé, la France pourrait être utile à l'Espagne, en Italie, grâce à son alliance avec l'Autriche et surtout en Amérique où elle pourrait l'aider à lutter contre l'Angleterre. L'alliance franco-espagnole fut donc renforcée et les couronnes des Bourbons s'unirent une fois de plus dans une politique étrangère commune afin de préparer les hostilités contre l'Angleterre. Par cette alliance Louis $\mathrm{XV}$ acceptait l'éventualité d'une guerre.

Ces considérations - l'aggravation des problèmes financiers et coloniaux de l'Angleterre, d'une part, et l'amélioration des relations diplomatiques entre la France et l'Espagne, d'autre part - constituaient des éléments importants du plan de guerre de Choiseul. Mais plus importantes encore, étaient la réorganisation générale des forces françaises sur le continent et dans

6 F.-P. Renault, "Etudes sur le Pacte de famille et la politique coloniale francaise, 1760-1790", Revue d'histoire des colonies françaises, dixième année (Premier semestre, 1922) : 47-48.

7 Blart, Les Rapports, op. cit., 77. 
les colonies et l'application d'une nouvelle stratégie de guerre, celle de diversion. Les colonies françaises devaient jouer un rôle capital dans la préparation des forces armées et la mise en application de cette stratégie. Leurs bases navales et militaires, renforcées par l'envoi de bataillons français, offraient un moyen pratique et direct d'attaquer les colonies anglaises. Les réformes administratives et économiques qu'on y avait apportées permettaient de suppléer à ce qui avait manqué pendant la guerre de Sept ans: l'efficacité et le soutien des colonies lors d'une lutte coloniale et maritime. Ces facteurs furent déterminants dans l'élaboration des plans de Choiseul et contribuèrent, toutes proportions gardées, à l'émergence d'une nouvelle ère qui atteignit son point culminant lors de la Guerre de l'Indépendance américaine.

Pour préparer sa guerre de revanche, Choiseul commença par réformer l'armée, la marine et l'artillerie. L'auteur de l'Essai Général de Tactique, le lieutenant général Jacques Guibert, apporte un exemple d'innovation militaire. D'après ce nouveau système, les troupes pouvaient manœuvrer plus librement en présence de l'ennemi au lieu d'être immobilisées dans une position prévue; des formations irrégulières furent introduites; les rangées de bataille ne devaient plus être rigoureusement droites, et les unités qui les composaient pouvaient être déployées dans un ordre opposé à l'ordre normal si cela était plus efficace. ${ }^{8}$ Le comte de Gribeauval chercha à améliorer l'aspect mécanique des fusils et de l'artillerie. En 1776, le lieutenant général Pierre Bourcet introduisit un corps d'officiers employé à la reconnaissance du pays qui devint un état-major spécial en 1770 . Choiseul pouvait aussi se vanter d'avoir presque doublé le nombre des vaisseaux de ligne français par ses réformes navales. Il écrivit à Louis XV en 1765 pour l'informer de ces dites réformes et de son plan d'attaque maritime:

Le calcul juste est que la France ne peut pas armer, pendant une guerre, plus de 80 vaisseaux de ligne et 40 frégates avec les bâtiments de moindre force, nécessaires à la suite des escadres et à la manutention des ports. J'appris ensuite que 80 vaisseaux et 40 frégates demandaient pour leur construction, leur rechange et leur fourniture tant de matière en magasin. Cette partie n'est pas difficile, car il n'y a que cinq matières nécessaires dans un arsenal de marine, le bois, le fer et cuivre, le chanvre et les toiles. En connaissant la quantité de chaque matière et les différentes sortes nécessaires à un bâtiment,

${ }^{8}$ Emile-G. Léonard, L'Armée et ses problèmes au XVIIIe siècle (Paris, Librairie Plon, 1958), 257-258. 
l'on sait ce qu'il faut dans un port pour le nombre de vaisseaux que l'on se propose d'y avoir, et la somme de dépense de ces vaisseaux pour la construction, l'entretien et l'armement. C'est d'après ces connaissances que je proposai, en 1764, à Votre Majesté d'avoir en quatre ans 60 vaisseaux de ligne, en cas que la guerre se déclare. Je distribuai ces 80 vaisseaux et les frégates fictivement dans les trois ports, et je calculai alors ce que Votre Majesté avait, et ce que j'avais, à faire. Vous aviez, Sire, au commencement de 1763, 44 vaisseaux de ligne tant bons que mauvais et 10 frégates. Vous avez à présent 63 vaisseaux de ligne et 31 frégates. J'ai presque doublé les forces de Votre Majesté, à la différence que l'été prochain, ces 63 vaisseaux seront tous en état d'aller à la mer, et en état de tous points, au lieu que, quand j'en ai pris le soin, ils étaient dans un désordre affreux. J'ai acquitté Votre Majesté en construisant tous les vaisseaux qui avaient été donnés en 1761 et 1762 et dont l'argent avait été dépensé alors en armements; et, en 1769 , les approvisionnements pour la construction des seize vaisseaux à faire pendant la guerre, seront dans les magasins, si l'on suit le plan que j'ai inventé, commencé d'exécuter, et que Votre Majesté a approuvé.9

La réforme de l'artillerie constituait un élément essentiel du programme de Choiseul. Il déplorait le peu d'améliorations techniques apportées au matériel d'artillerie depuis la fin du règne de Louis XIV. Sa réforme favorisa la coordination des forces de l'artillerie maritime et terrestre et conduisit à la formation de brigades. On allégea le poids des fusils en raccourcissant leurs canons et cette innovation permit une visée plus facile et plus précise. Les canons furent améliorés et standardisés dans tous les ports, sous la direction de Moroguer et de Martil, experts en artillerie. ${ }^{10}$

De plus, Choiseul purgea et remania le corps des officiers. Mentionnons à ce sujet que les pouvoirs des intendants établis par Colbert en vertu de l'Ordonnance de 1689 ainsi que les pouvoirs de la hiérarchie administrative connue sous le nom de la plume, avaient été plus importants que ceux accordés à l'exécutif, l'épée. "Le corps de plume était l'objet de l'animosité de l'épée", écrivait Choiseul en faisant observer au roi que la plume avait joui de privilèges importants depuis l'Ordonnance de Colbert, et

9 Choiseul, Mémoire, op. cit., 17-18. Voir aussi: Mémoire sur les forces de mer et de terre de la France et l'usage qu'on pourrait en faire en cas d'une guerre avec l'Angleterre, Archives d'Affaires Etrangères, Correspondance politique, Espagne, vol. 550: fols. 495-497 (Abréviation utilisée ci-dessous: A.E.C.P.).

10 Choiseul, Mémoire, op. cit., 18. 
qu'elle avait négligé l'intérêt du gouvernement, mal représenté et maltraité les officiers, et recherché uniquement son propre intérêt. ${ }^{11}$ Il ajoutait qu'il s'était employé à corriger cette situation en remédiant aux abus et en améliorant l'efficacité de l'armée et de la marine.

Le programme des réformes militaires de Choiseul englobait les colonies où des changements radicaux devaient permettre aux régiments coloniaux de se préparer à la guerre. Par l'Ordonnance du 24 mars 1763, le principal ministre de Louis XV réforma l'administration coloniale, fit renforcer et accroître la défense des colonies par la construction de nouvelles fortifications, par le stockage de dépôts d'armes et de munitions en approvisionnement suffisant. Il fit remplacer les anciennes milices coloniales par des troupes françaises régulières en provenance de la Métropole et, plus tard, il dut remettre sur pied les milices locales pour renforcer les régiments français. ${ }^{12}$ Dans son Mémoire au roi, Choiseul rapportait qu'il était en train d'instaurer un système européen en Amérique sous la direction d'administrateurs compétents investis de l'autorité nécessaire pour faire des réformes supplémentaires. ${ }^{13}$ Choiseul ajoutait:

Ces deux points sont essentiels à la défense de l'Amérique. Je n'entrerai point dans le détail de ce qu'il y a à faire dans les colonies, ce détail seul demanderait des volumes, mais je dirai en un mot à Votre Majesté que, si Elle avait la guerre contre les Anglais, il serait instant, au moment qu'on l'envisagerait, de faire passer en Amérique vingt-quatre bataillons qui trouveraient dans les îles ce qui leur serait nécessaire, resteraient pendant toute la guerre en Amérique, et seraient alimentés tant en vivres qu'en munitions par les escadres de Votre Majesté dans cette partie du monde. ${ }^{14}$

Ecrivant aux comtes de Belzunce et de Clugny, respectivement gouverneur lieutenant général et intendant des Iles sous le Vent, Choiseul leur mentionna que le roi avait sanctionné des mesures législatives pour remédier aux lacunes et pour augmenter les forces militaires de façon à ce que les colonies fussent prêtes à

11 Ibid. 340-357.

12 Ordonnance de mars 1763, Archives Nationales, Col. A., 8: fols.

${ }_{13}$ Choiseul, Mémoire, op. cit., 19. Le Comte d'Estaing fut envoyé à Saint-Domingue; le Comte d'Ennery à la Martinique, le Comte de Nolivas à la Guadeloupe et le Comte de Fiedmond en Guyane française. Voir aussi le Mémoire du Roi, Cayenne, Année 1764, Archives Nationales, Minutes des Dépêches et Ordres du Roi, Col. B. 119: fols. 20-35 (Abréviation utilisée ci-dessous: A.N., Minutes des Dépêches).

14 Ibid.: 20. 
temps pour la guerre. ${ }^{15}$ Lorsque le comte d'Estaing remplaça Belzunce, il fut avisé que la première responsabilité du gouverneur était d'ordre militaire. "Son principal objet, en arrivant, doit être premièrement de distribuer dans les principaux quartiers de colonie... les six bataillons qui resteront en garnison à Saint-Domingue ${ }^{16}$." Arrivant en 1768 avec une flotte importante, Estaing mit sur pied une légion avec 400 recrues de l'Ile de Ré, centre de recrutement pour les colonies établi par Choiseul. Entre 1768 et 1770, Saint-Domingue et d'autres colonies antillaises reçurent un supplément considérable de troupes et de renforts. C'est ainsi, par exemple, qu'en 1768 furent expédiés à Saint-Domingue les articles suivants: ${ }^{17}$

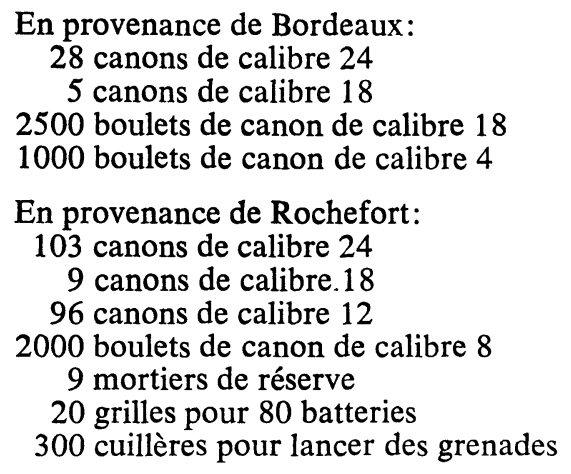

En provenance de Brest:

1790 boulets ramés de calibre 24

1290 boulets ramés de calibre 18

500 boulets ramés de calibre 12

1000 boulets ramés de calibre 8

500 boulets ramés de calibre 4
En provenance de Toulon:
500 quintaux de poudre
8 canons de calibre 4
3500 boulets de canon de calibre 24
30 mortiers de réserve
1680 bombes
200 grenades à main

15 Mémoire $d u$ Roi, Isles sous le Vent, Année 1763, A.N., Minutes des Dépêches, Col. B. 116: fols. 142-143.

16 Ibid., vol. 119: fols. 20-35. Voir aussi Envoy d'un Mémoire concernant la police et la défense des colonies en paix comme en guerre, Mai 1764, A.N., Minutes des Dépêches, ibid.: fol. 113.

17 Ordonnance du 13 janvier 1768, A.N., Minutes des Dépêches, Col. B. 130 : fols. $71-72$. 


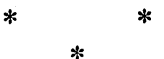

La Martinique et la Guadeloupe firent l'objet d'un renforcement des préparatifs militaires, semblable à celui de SaintDomingue. Des bases militaires furent établies sur les deux îles pour abriter jusqu'à vingt-quatre bataillons. Cependant, la Martinique joua un rôle plus important que celui de la Guadeloupe, à cause de sa position stratégique et de la situation avantageuse de ses ports. Elle devint la principale base navale pour les vaisseaux continentaux, notamment pendant la guerre de l'Indépendance américaine. Sous la direction du comte de Rochemore et d'une équipe d'ingénieurs, la Martinique fut pourvue de fortifications supplémentaires et, en général, les défenses côtières furent améliorées. Les troupes françaises régulières, en vertu de l'Ordonnance générale du 24 mars 1763, remplacèrent d'abord les milices coloniales de la Martinique et de la Guadeloupe; mais, à compter de 1768 , ces dernières furent à nouveau mises sur pied pour renforcer la défense nationale. ${ }^{18}$

Choiseul, a-t-on dit précédemment, avait conçu son programme de réformes en vue de soutenir une guerre maritime et coloniale, plutôt qu'une guerre continentale. Il s'attendait à ce que les hostilités éclatent sur le continent mais il espérait qu'avec l'aide espagnole et le soutien des régiments coloniaux, elles seraient détournées vers les colonies. Ses prévisions se fondaient sur l'anticipation du retour, à la tête du ministère britannique, de William Pitt, comte de Chatham, qui avait démissionné de son poste de secrétaire d'Etat en 1761 . Face à l'éventualité du retour au pouvoir de ce chef d'Etat connu pour sa politique conquérante, Choiseul prévoyait que les hostilités anglo-françaises éclateraient à nouveau sous la forme d'une guerre continentale. Dans ce cas, la France pourrait contre-attaquer par une guerre de diversion contre l'Angleterre.

En élaborant ses plans pour une guerre de diversion, Choiseul avait soigneusement examiné les positions diplomatiques respectives des diverses puissances européennes, de façon à en déterminer le jeu vis-à-vis de la France et de l'Angleterre. C'est ainsi qu'il mit Louis XV en garde contre les ambitions de la Maison d'Autriche en Italie. Ces visées autrichiennes risquaient de perturber la paix établie par le Traité de Paris, ce que la France devait éviter à tout prix pour se donner le temps de se préparer à la guerre. Choiseul remarquait à ce sujet:

18 De plus amples détails concernant l'importance des Antilles françaises sont donnés plus loin. 
En Italie, la tranquillité ne peut être troublée que par la Maison d'Autriche, soit qu'elle porte son ambition à la conquête du royaume de Naples, soit qu'étant en guerre contre la France, Votre Majesté songeât à faire une diversion contre elle dans ses Etats d'Italie. Dans les deux cas, le Roi de Sardaigne est nécessaire, et je suis d'avis qu'il faut, pour ces objets, ménager soigneusement la cour de Turin. Tant que Votre Majesté sera unie à la cour de Vienne, l'Italie sera tranquille, mais cette union, comme je l'ai expliqué plus haut, peut cesser; il faut même, pour la soutenir, envisager chaque jour sa fin et la guerre. ${ }^{19}$

Les préoccupations du ministre de Louis $\mathrm{XV}$ en regard de la position de l'Italie dans le jeu des affaires européennes provenaient aussi des tentatives faites par la Cour de Turin pour établir des relations diplomatiques avec le gouvernement britannique:

Il n'y a à prévoir pour le succès que l'attachement enraciné de la cour de Turin pour celle de Londres, attachement du Plaisantin, sans lequel la cour de Turin, il n'en faut pas douter, aurait resserré ses liens avec Londres; au lieu que la confiance dans Votre Majesté, qui a été solidement acquise par la netteté et la bonne foi de cet arrangement, a arrêté les menées de l'Angleterre à Turin et nous a mis en mesure de faire valoir puissamment l'intérêt du Roi de Sardaigne en faveur de la France, si les circonstances portent la guerre en Italie. ${ }^{20}$

Ainsi Choiseul désirait-il améliorer les relations avec Turin et la Sardaigne dans le but de contre-balancer toute influence anglaise possible en Italie, et de maintenir des relations amicales entre la France et l'Autriche.

Le cas de la Hollande n'était pas sans préoccuper le ministre français: "Elle est intérieurement aussi ennemie de la France que l'Angleterre, confiait-il au roi, et n'est retenue dans son animosité que par l'avidité des gains que cet Etat fait par la neutralité." Dans son plan d'une guerre continentale de diversion, Choiseul envisageait d'attaquer la Hollande au moment où l'Espagne attaquerait le Portugal, ainsi qu'en témoigne son Mémoire:

J'ose, dis-je, avancer à Votre Majesté que, dans ce cas, je lui conseillerais d'agir vis-à-vis de la Hollande comme l'Espagne devrait agir vis-à-vis du Portugal. A mon avis, je porte-

19 Choiseul, Mémoire, op. cit., 8.

20 Ibid. 
rais la guerre dans les Sept Provinces, qui me serviraient, en cas de succès, de nantissement aux pertes que Votre Majesté pourrait faire en Amérique. ${ }^{21}$

Choiseul assura Louis XV qu'une fois la guerre continentale commencée, des réformes et des préparatifs militaires raisonnables ayant été réalisés, la France pourrait occuper les Anglais en Europe pendant que l'armée et la marine coloniales françaises envahiraient les colonies anglaises du Nouveau-Monde. Il avança qu'avec l'avantage fourni par leur position unique, les forces militaires de la Martinique, de la Guadeloupe et de SainteLucie étaient assez considérables pour permettre une invasion des colonies anglaises des Indes occidentales. En plus, l'Espagne, alliée à la France par le Pacte de Famille, devait jouer un rôle important dans la guerre de diversion envisagée, en renforçant les forces françaises tant en Europe que dans les Antilles. En engageant la guerre contre le Portugal, comme en 1762, l'Espagne diviserait les forces anglaises en Europe. L'Angleterre serait alors incapable d'envoyer des troupes en Amérique du Nord et dans les Antilles, et la route des colonies anglaises serait ainsi ouverte aux régiments coloniaux franco-espagnols.

C'est pourquoi la guerre contre le Portugal sera toujours, dans tous les cas, une guerre utile à Votre Majesté, quelque événement qui en résulte... Je ne sais si l'on pourra parvenir à reculer jusqu'à cette époque le malheur de la guerre, surtout si M. Pitt rentre en place, mais je crois qu'il faut employer tous ses talents pour y parvenir. ${ }^{22}$

La guerre projetée contre le Portugal serait doublée d'une attaque contre le Brésil par les forces coloniales appartenant aux couronnes bourboniennes. Ainsi arrêterait-on la formation d'un empire portugais qui pourrait devenir menaçant pour les colonies espagnoles d'Amérique du Sud et pour les possessions antillaises demeurées sous le contrôle de la France après la guerre de Sept ans.

Si l'Angleterre attaque la France seul... l'Espagne ferait peut-être ensuite une attaque de diversion contre le Portugal et peut-être ensuite l'Irlande... l'Espagne ne doit pas annexer le Portugal car le Roi partirait pour le Brésil et ruinerait les possessions espagnoles en Amérique. La guerre de diversion doit durer 4 ou 5 ans. ${ }^{23}$

${ }^{21}$ Ibid., 9.

22 Ibid.

23 Mémoire des questions préalables à résoudre entre les 2 cours avant d'arrester définitivement un projet sur la guerre de Portugal, Bibliothèque Nationale, Papiers de l'abbé Béliardi, Mss. Fr. 10770: fols. 43-44 (Abréviation utilisée ci-dessous: B.N., P.B.). 
Des renforts supplémentaires, envoyés de la base militaire de Guyane, viendraient à l'aide des régiments français, contre les Portugais. La position stratégique de la Guyane, dont il sera question plus loin, fut déterminante dans le choix opérationnel de Choiseul.

Le plan de guerre que Choiseul élabora dans son Mémoire de 1765 avait été conçu dès 1763 dans un écrit intitulé Réflexions sur une guerre particulière où l'on peut lire:

L'Angleterre s'est arrangée auparavant pour empêcher la France d'avoir une Marine puissante et la faire combattre sur terre en Europe ... Mais la France fera désormais une guerre de mer. ${ }^{24}$

Ces réflexions prévoyaient une attaque anglaise, contre la France, sur le continent. On y affirmait que Choiseul avait le dessein de contre-attaquer, sur mer, avec quarante vaisseaux de ligne ancrés à Brest et à Rochefort et vingt vaisseaux de ligne ancrés à Toulon. Trois compagnies de troupes régulières sur chaque vaisseau de ligne augmenteraient encore la défense française. On espérait que la contre-attaque française serait si redoutable que les forces britanniques se verraient obligées de se concentrer sur la côte de Bretagne, pour surveiller la Manche, dans le golfe de Gascogne et dans la Méditerranée. Comme le précise le Mémoire de 1765, une telle concentration de la marine britannique dans les eaux européennes priverait les colonies anglaises des Indes occidentales de défenses adéquates et laisserait aux forces françaises des Antilles la liberté de frapper à volonté. On y comptait alors à peu près vingt-quatre bataillons, en excluant une armée de 4,000 hommes postés à Saint-Domingue et une armée plus petite venue de Guyane. ${ }^{25}$ L'escadre française avait le choix d'une base naturelle au Môle Saint-Nicolas (Haïti) ou au Cap Tiburon (Saint-Domingue). En plus, à Brest, la flotte française empêcherait les forces anglaises cantonnées en Europe de se porter à l'aide de leurs possessions antillaises. Le plan prévoyait que cette flotte serait aidée par les troupes françaises rassemblées en Flandre, en Bretagne et à Ferrol en Espagne, et que si les Anglais essayaient de déplacer leurs forces d'Europe vers les colonies, deux escadres supplémentaires de quinze vaisseaux de lignes pourraient être envoyées à Saint-Domingue avec cent vingt compagnies. fol. 63 .

24 Papiers de l'abbé Béliardi, Bibliothèque Nationale, Mss Fr. 10770 :

25 Voir note no 13 , ci-dessus. 
D'après les Réflexions, les Français cherchaient à couper les approvisionnements de guerre dans les colonies anglaises en Amérique (à Providence, à Saint-Augustin et aux Bahamas) et à détruire le commerce anglais avec Terre-Neuve, le Québec, la Floride et la Jamaïque. On prévoyait une campagne victorieuse à ces endroits stratégiques en prenant pour acquit que les forces coloniales françaises seraient prêtes à porter le grand coup avant que l'Angleterre ne déclare la guerre. ${ }^{26}$

Les Réflexions et le Mémoire postulaient également un début de guerre continentale, suivi par une guerre coloniale et maritime. Le projet original de 1763 fut donc maintenu, mais le Mémoire de 1765 contenait un plan d'attaque plus élaboré qui s'appuyait spécifiquement sur une guerre de diversion. Tels furent les éléments constitutifs des desseins belliqueux du ministre français entre 1763 et 1770 . A cet effet Choiseul déclara dans un autre Mémoire :

Ce plan est grand, je le crois utile; il demande à être expliqué, et, si je ne suis pas mort quand le cas arrivera, et que Votre Majesté s'en souvienne, dans quelque lieu que je me trouve, j'en donnerai à Votre Majesté les explications si Elle les désire. C'était le projet que je comptais lui proposer pour 1763, si la paix ne s'était pas faite. ${ }^{27}$

Il concluait que c'était un tel projet qui l'avait incité à instaurer un programme de colonisation.

L'expédition de Kourou fut le plus remarquable exemple de la politique d'expansion de Choiseul qui cherchait à établir de nouvelles bases militaires en Guyane française.

Aucune colonie n'offre à la France autant d'avantages que la Guyane, pour remplacer celles que la guerre nous a enlevées, pour assurer la défense de celles qui restent et pour nous procurer des moyens d'attaque et de diversion contre nos ennemis naturels ou contre leurs alliés, si la guerre se rallume un jour entre eux et nous. ${ }^{28}$

26 Réflexions sur une guerre particulière entre la France et la GrandeBretagne, A.E.C.P., France, M. et D., vol. 574: fol. 143 (Abréviation utilisée ci-dessous: Réflexions).

27 Choiseul, Mémoire, op. cit., 9.

28 Copie tirée d'un projet d'établissement d'une nouvelle colonie dans la Guyane, 1763, Bibliothèque Nationale, Fr. 6244: fol. 83. Voir aussi H. Ternaux-Compans, Notice historique sur la Guyane française (Paris, Société d'études pour la colonisation de la Guyane française, 1843), et M. Artur, Histoire manuscrite des colonies françaises de la Guyane (Bibliothèque Nationale, N.A. 2571-2572: fol. 781). 
C'est ce que confirme un article de Jean Chaia qui écrit:

Peut-être n'était-ce même pas de revanche qu'il s'agissait, mais de défense. De toute façon il fallait se tenir prêt, d'autant que l'Angleterre commençait à rencontrer dans ses colonies d'Amérique de sérieuses difficultés. ${ }^{29}$

Ainsi peut-on soutenir que la colonisation de la Guyane fut entreprise dans le but explicite de renforcer les forces militaires françaises dans les Antilles. Louis XV lui-même exposa ses vues à ce sujet dans un Mémoire à Turgot, nommé gouverneur de la colonie française de Kourou et gouverneur général de la Guyane:

Le sieur Chevalier Turgot, y lit-on, ne doit point perdre de vue que l'intention de Sa Majesté est d'établir la nouvelle colonie en Blancs, tant parce que cette population est plus compatible avec les vues de justice et d'humanité qui animent Sa Majesté, que parce qu'elle est plus propre à procurer à un Etat un degré de force capable d'imposer. Ce système de population est d'autant plus nécessaire, que les Anglais n'ont fait leurs conquêtes, dans la dernière guerre, que par le moyen de leurs colonies septentrionales, qui sont presque uniquement peuplées de Blancs, et que, formant actuellement, à la Dominique, une colonie dont les noirs sont exclus, il est aisé de voir qu'ils méditent de faire, par son moyen, la conquête de la Martinique et de la Guadeloupe à la première occasion de guerre. S.M. ne peut donc leur opposer des forces capables de balancer les leurs qu'en peuplant aussi de Blancs la colonie de la Guyane, laquelle ... est très favorablement placée pour leur porter des secours, et même pour agir offensivement contre les îles Anglaises. En conséquence elle charge le M. Chevalier Turgot de s'occuper avec soin de connaître les divers genres de culture qui conviennent spécialement aux Blancs, de les encourager et d'inspirer les mêmes vues à ceux qui seront chargés de commander en son absence. ${ }^{30}$

L'implantation de nouvelles colonies et l'établissement de bases militaires représentaient des éléments essentiels dans l'élaboration d'une nouvelle stratégie offensive de la France après le Traité de Paris, ainsi que le démontre le Mémoire de 1765:

29 Jean Chaia, "Echec d'une tentative de colonisation de la Guyane au XVIIIe siècle", Biologie Médicale, XLVII (avril 1958): xiii. Voir aussi Paul Henry, Sur une tentative de colonisation en Guyane (thèse pour le Doctorat en Médecine: Faculté de Médecine de Paris, 1940), 11.

30 Mémoire du Roi pour servir d'Instructions au Sieur Chevalier Turgot Gouverneur lieutenant général de la Guyane, le 31 août 1764, A.N., Col. C. 14, vol. 27: fols. 46-47. 
C'est d'après ce plan que nous préparons les possessions de Votre Majesté dans cette partie du monde, et je crois que ce plan, qui doit réussir malgré les retardements qu'il a éprouvés par mes fautes, est le seul qui puisse assurer à Votre Majesté ses possessions et les mettre en état de menacer celles de ses ennemis et même d'avoir de succès en Amérique..$^{31}$

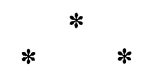

Nous avons essayé de montrer dans cette étude toute l'importance stratégique des colonies antillaises françaises dans la préparation d'une guerre de revanche contre l'Angleterre après 1763. Convaincu que Louis XV n'approuverait pas de si tôt une nouvelle ouverture des hostilités, Choiseul en profita pour trouver dans les colonies des endroits stratégiques et prévoir l'utilisation des ressources coloniales tout en élaborant un plan de guerre de diversion en Europe. Dans la conjoncture révolutionnaire qui se développait en Amérique du Nord et étant donné la préparation adéquate de toutes les forces françaises de terre et de mer, son plan pour une guerre coloniale et maritime paraissait valable.

Mais Choiseul n'eut pas la possibilité de réaliser ses desseins belliqueux alors qu'il était en fonction, puisqu'il fut renvoyé en 1770. Ce fut son successeur, le comte de Vergennes, qui allait profiter de la Révolution américaine pour mettre en œuvre le plan de guerre de revanche contre l'Angleterre avec la participation des Antilles.

Traduction de Christine N. GaZAGNeS

Université du Nebraska

${ }^{31}$ Choiseul, Mémoire, op. cit., 20. 